\title{
HACIA UNA DEFINICIÓN DE LO "INTER" DE LA INTERACCIÓN COMUNICATIVA INTERCULTURAL
}

\author{
TOWARDS A DEFINITION OF "INTER" IN THE INTERCULTURAL COMMUNICATIVE \\ INTERACTION
}

\author{
Enrique Huelva Unternbäumen \\ Universidad de Brasilia - Brasil \\ enriquehuelva@gmail.com
}

\begin{abstract}
Reflexionamos en este breve texto sobre la naturaleza de la interacción comunicativa intercultural. Rechazamos la posibilidad de caracterizar el espacio creado en encuentros interculturales desde una óptica subjetivista, esto es, como la simple suma de las actividades subjetivas de los interactuantes involucrados. Al mismos tiempo, argüimos que ese espacio no está estructurado, de ante mano, ni por elementos de una pragmática universal ni por tipicidades comunicativas de ninguna de la culturas que entran en contacto. Como alternativa, proponemos concebir la interacción comunicativa intercultural como una entidad sui generis que emerge autogenéticamente.
\end{abstract}

Palabras clave: Comunicación Intercultural; Interacción; Pragmática Transcultural; Autogénesis.

Keywords: Intercultural Communication; Interaction; Transcultural Pragmatics; Autogenesis.

In this short text, we reflect on the nature of intercultural communicative interaction. We reject the possibility of characterizing the space created in intercultural encounters from a subjectivist angle, i.e., as a simple sum of the subjective activities of the interactors involved. At the same time, we argue that this space is not structured in advance, either by elements of a universal pragmatics or by typical communication patterns of any of the cultures that enter into contact. As an alternative, we propose conceiving of intercultural communicative interaction as an entity sui generis that emerges autogenetically.

(Recibido: 5/1/16; Aceptado: 11/2/16)

1.

Estamos en la era científica de los prefijos, por lo menos en las Humanidades. INTER, TRANS, MULTI, POS, HIPER, para nombrar talvez los representantes más prominentes, han dejado hace tiempo de contentarse con el papel que la lengua normalmente les atribuía, a saber, el de expresar una cualidad secundaria del referente del sustantivo que prefijan. Su ambición es mayor y el papel pretendido diferente. 
Lejos de denotar tan sólo un mero atributo del sujeto, del cuerpo, de la acción, del texto, de la cultura o de la modernidad, los prefijos sustraen y se apoderan de la capacidad referencial del sustantivo, se sustantivan, invitándonos o quizás mejor, desafiándonos a imaginar nuevos espacios epistemológicos y objetos con esencias propias, resultantes de diferencias, duplicaciones, pliegues, despliegues, repliegues..., complejidades, que, frecuentemente, reivindican una primacía y originalidad frente a los objetos designados por los sustantivos que los subsiguen.

El título de las reflexiones que les propongo recoge este desafío en el caso de INTER en la interacción comunicativa intercultural. Tomarse este desafío en serio significa, antes de todo y antes que nada, reconocer la existencia de un espacio sui generis entre las culturas, así como de un espacio entre los individuos de diferentes culturas que interactúan comunicativamente.

\section{2.}

Empecemos reflexionando sobre la dimensión individual. Reivindicar la existencia de un espacio entre los interactuantes supone que lo que está y lo que sucede en este espacio no pertenece a ninguno de ellos y, ni siquiera, a todos ellos juntos. El espacio intermediario es, como apunta Waldendels (2006: 110), tierra de nadie (Niemandsland). Lo que sucede en ella no es reducible ni a las acciones individuales de los interactuantes, ni a la suma de las mismas. Siempre es algo más y, sobre todo, algo distinto. El individuo es incapaz de prever o controlar totalmente a través de sus acciones intencionales qué dirección tomarán los acontecimientos. En el mejor de los casos - quizás incluso en el más normal de ellos - escogerá un enunciado que se ajuste a lo ocurrido en la comunicación hasta aquel momento, procurando combinarlo con el enunciado precedente de modo que juntos formen un texto coherente. Al mismo tiempo, sin embargo, nunca sabrá (y nunca sabremos) qué reacciones producirá su enunciado en el interlocutor: te pregunto la hora y me miras callada con ademán de desprecio o me dices que demasiado tarde o que me compre un reloj o que son las doce y cuarto o quizás otra cosa o a lo mejor nada. En la tierra de nadie hay actividad subjetiva, pero no pertenece ni su en totalidad ni en su esencia a ningún sujeto.

Una salida para determinar lo que ocurre en este 'espacio de nadie' podría ser imaginarnos que, de ante mano, ya nos pertenece a todos. En este caso, en vez de un espacio de nadie sería un espacio de nadie en particular, esto es, un espacio colectivo, un espacio de todos. Es lo que ocurre cuando postulamos que las actividades comunicativas individuales se realizan siguiendo pautas universales que se manifiestan, dependiendo del marco teórico, en la forma de principios y máximas conversacionales, de tipos y familias de actos de habla o de contextos pragmáticos típicos e idealizados. Estos elementos universalmente válidos existirían antes e independientemente de cualquier actividad comunicativa individual y servirían de guía general para la realización de las mismas. Serían, para continuar con la metáfora, como caminos preexistentes que habría que tomar para adentrarnos en ese espacio ahora ya y desde siempre de todos y que garantizarían la realización exitosa de nuestros encuentros comunicativos en él.

Contra esta visión de una pragmática universal existen, sin embargo, objeciones importantes, tanto empíricas como teóricas. Respecto a las primeras, cabe destacar que los estudios en Pragmática Transcultural realizados en las últimas décadas demuestran, sin dejar ningún género de dudas, que existe un relativismo inter- e incluso intracultural significativo de las actividades comunicativas, incluso de las más simples y habituales de nuestro cotidiano (cf. Wierzbicka 2003). No todo el mundo realiza las mismas actividades comunicativas, y, si las realiza, no lo hace con la misma frecuencia ni de la misma manera. Yo no ordeno que se vayan los espíritus, como lo hacen los miembros de la cultura Kamaiurá durante la ceremonia del Kwaryp, del mismo modo que un musulmán creyente no brinda con una copa de vino a la salud de los otros. 
Y cuando me veo en la situación de tener que comentar críticamente una tesis durante un tribunal de doctorado sé que en Brasil lo tengo que hacer suavemente y con preámbulos positivos, mientras que en Alemania de forma directa y sin rodeos, a no ser que quiera que en el primer caso me tachen de bruto y en el segundo de poco objetivo.

Si proseguimos esta discusión en un nivel más teórico nos toparemos fácilmente con más argumentos que refuerzan la noción de un relativismo cultural de la acción comunicativa. ¿Qué estatus teórico debemos atribuir, por ejemplo, a las funciones esenciales de la acción comunicativa propuestas por la Teoría de los Actos de Habla y que le sirven para concebir una clasificación universal de los mismos? Tenemos, básicamente, dos opciones. Podemos entenderlos como universales funcionales a priori. Si optamos por ello, nos veremos obligados a explicar cómo es posible que existan funciones básicas de la interacción social (como, por ejemplo, prometer, ordenar, declarar nuestro amor o expresar nuestro odio) sin que existiera antes ya la sociedad y las actividades comunicativas que la generan. Nos resta la alternativa de concebirlas como universales empíricos, esto es, como funciones comunicativas que, demostradamente, se encuentran en todas o en la mayoría de las culturas y lenguas del mundo. Pero esta opción nada más hace que reafirmar su origen comunicativo y, en consecuencia, su particularismo cultural. No sería difícil trazar una reflexión semejante sobre el estatus de máximas o principios para los que se reivindica una vigencia universal como reguladores de nuestro comportamiento comunicativo. ¿Cómo afirmar que existan antes de la comunicación? Y, al contrario: ¿Cómo es posible que exista comunicación sin la necesidad de que ésta esté regulada por los efectos de principios y máximas que aspiran a ser la condición de su posibilidad? Y ahora, ¿qué hacemos con esta paradoja? Valga de momento la afirmación de que las funciones comunicativas esenciales, las máximas y los principios no son caminos que determinan la dirección de nuestros pasos por el espacio de nadie, sino que, como dice el poeta, más bien lo que sucede es que se hace camino al andar.

\section{3.}

Dijimos al inicio de nuestras reflexiones que para caracterizar adecuadamente lo INTER deberíamos llevar en cuenta también la existencia de un espacio sui generis entre las culturas y no sólo entre los individuos. Vamos a intentarlo.

La Antropología, la Etnografía del Habla o de la Comunicación y, más recientemente, la Pragmática Transcultural dejan amplia constancia de que la interacción comunicativa no puede ser concebida como un intercambio de unidades comunicativas mínimas, independientes o aisladas las unas de las otras, entre el hablante y el oyente. Más bien, al comunicarnos, seguimos una cierta tipicidad que establece, convencionalmente, que para realizar una determinada actividad comunicativa hemos de producir una secuencia de actos de habla interrelacionados. Lo que en un determinado contexto pragmático puede ser dicho para realizar una función comunicativa y el orden en el que se pueden decir las cosas están altamente convencionalizados. Existen, para así decirlo, reglas para el habla o reglas para la realización de actos de habla que determinan cómo, cuándo, quién, en qué orden, para quién, mediante qué elementos de la lengua, etc. se puede realizar una determinada actividad comunicativa.

Una observación adicional importante para nuestros objetivos es que estas reglas del habla varían entre las lenguas, entre comunidades culturales que usan la misma lengua e incluso en el seno de una misma comunidad lingüística y cultural (por ejemplo, en subculturas). No se pide un café en el Café das Letras de la Universidad de Brasilia de la misma forma que se pide en el Café de la Candelaria en Santiago de Chile o en el Café Gijón de Madrid. 
Y créanme, queridos estudiantes de español, si lo intentan pedir como lo enseñan los manuales didácticos de ELE, no se lo van a servir nunca.

La Pragmática Transcultural se refiere al relativismo cultural de la tipicidad comunicativa o de las formas de interacción mediante el uso del término cultural scripts, guiones culturales (cf. Goddard 2006). Poseer competencia comunicativa significa, desde la perspectiva que establece este concepto, conocer y saber usar adecuadamente los guiones culturales propios de una determinada cultura.

La constatación de un relativismo cultural de la tipicidad comunicativa es, sin duda, una condición esencial para la posibilidad de caracterizar lo INTER de la interacción comunicativa intercultural, puesto que define el escenario inicial del encuentro intercultural. Es una condición para la posibilidad, un prerrequisito, un punto de partida, pero ciertamente no es todavía la caracterización en sí (cf. Spencer-Oatey\&Kotthoff 2007: 1-2). Sabemos ahora que llegamos al encuentro intercultural pertrechados de nuestro respectivo instrumentario de guiones culturales. Pero, ¿qué sucede a partir de ahí? ¿Cómo se desarrolla el encuentro intercultural en sí? ¿Cómo entran en juego los respectivos guiones culturales?

Sin duda hemos de estimar que sus posibilidades de entrar en juego y su efectividad son mucho más limitadas que en los encuentros mono culturales. En estos, el guion es fácilmente identificado como conocimiento compartido, como trazado común que establece pautas de comportamiento y expectativas seguras para nuestros movimientos en el espacio interactivo. En los encuentros interculturales, por el contrario, la diversidad de los guiones - diversidad conocida, sospechada o insospechada pero que rápidamente se nos sobreviene y nos sorprende - produce incertezas, ambigüedades, trazados paralelos, múltiples, diáfanos, dudas para la proyección del próximo paso, incertidumbre acerca de mi ubicación y la tuya. Ninguno de los scripts consigue imponer unívocamente una topografía única con trazados claros para nuestros movimientos. Los desenlaces son variados. Desde el boqueo mutuo producido por la falta de identificación del guion iniciado o por la proyección de guiones culturales contradictorios o contrapuestos, pasando por reconocimientos parciales o tentativos, hasta, en el mejor de los casos, pero quizás no en el más frecuente, la coincidencia o la negociación y el acuerdo de una hoja de ruta común.

En todo caso, tenemos siempre una supresión de la seguridad que emana del saberse partícipe de una misma tipicidad y de volver a pisar caminos ya transitados. Baja el nivel de una intersubjetividad tácita y apriorística (hursserliana, podríamos decir) y sube la necesidad de construirla, en la medida de lo posible, en el propio encuentro (tornándose una intersubjetividad más cercana a la propuesta por Alfred Schütz), a no ser que queramos correr el riesgo de que nos topemos sin encontrarnos o de que, sin darnos cuenta, creamos que nos hemos encontrado pero, en realidad, hemos pasado de largo. ${ }^{1}$

La profesora brasileña regresa al Departamento después de comprar su merienda. En el pasillo, cerca de la entrada, encuentra a su colega suizo. Tras saludarse, se le sobrepone situacionalmente la necesidad de expresar cortesía mediante el guion cultural del ofrecimiento figurado de lo que se está comiendo o se va a comer: "aceita?, servido?" Para su sorpresa, el colega suizo coge la merienda, agradece con entusiasmo y se la come. Ella se queda sin merienda, con hambre y con la impresión de que el suizo es bastante maleducado o de que estaba talvez muy hambriento. En el pasillo del Departamento, el profesor suizo ve acercarse a su colega brasileña.

\footnotetext{
${ }^{1}$ Sobre estas dos definiciones del concepto de la intersubjetividad, cf. Duranti (2010). Se recomienda también la lectura de Gallagher (2012: 182-204) para una presentación más detallada del concepto fenomenológico de la intersubjetividad.
} 
Tras saludarse, y para su sorpresa, ésta le ofrece amablemente algo para comer: "aceita, servido?". Aunque en realidad no tenía hambre y ni siquiera le gustase lo que su colega brasileña le estaba ofreciendo, al profesor suizo le sobrevino la obligación de aceptar la oferta, puesto que rechazarla supondría correr el riesgo de ser considerado descortés. Y así, sin hambre y sin apetito, pero con educación y gratitud, tuvo que comerse lo que no quería.

Volvemos a donde ya estábamos, aunque ahora con más equipaje: en la tierra de nadie hay actividad subjetiva, pero no pertenece ni su en totalidad ni en su esencia a ningún sujeto. En la tierra de nadie hay tipicidades culturales, hay guiones culturales, formas culturalmente específicas de interactuar, pero ninguna de ellas es dueña ni de su totalidad ni de su esencia.

\section{4.}

Si ni el sujeto ni la cultura son capaces de garantizarnos a priori una unidad de lo INTER, ¿qué nos resta? Podríamos pensar en multiplicidades. El espacio de la interacción intercultural estaría formado, de acuerdo con esta alternativa, por varios sujetos y sus respectivas actividades individuales y por varias tipicidades culturales. Pero esta opción significaría, como es obvio, negar justamente lo INTER. En su lugar tendríamos, como bien apunta Waldenfels (2006: 117-118), un conjunto de nómadas, de eremitas que deambularían por el mismo espacio pero por diferentes caminos sin encontrarse.

$\mathrm{Si}$, por el contrario, continuamos apostando por la posibilidad del encuentro, lo que nos resta es concebir lo INTER como un proceso de autogénesis, de sociogénesis, como lo propone la Sociología Fenomenológica (Schütz 1993) o de autopoiesis, como lo postula la Teoría del Sistema de Luhmann (1997: 60-120), fundamentándose en los pensadores chilenos Humberto Maturana y Francisco Varela. ${ }^{2}$

Como es bien sabido, la Sociología Fenomenológica de Alfred Schütz (1993), Peter Berger y Thomas Luckmann (1987) - para nombrar a sus representantes más destacados - sitúa el origen de la realidad social en la propia acción social, esto es, en la interacción. Nuestras acciones sociales no se orientan tan sólo a un sentido y a una realidad social preestablecidos, sino que en ellas y a través de ellas se crea ese sentido y esa realidad social. Decimos que la interacción es el origen del sentido, pero, al mismo tiempo hemos de puntualizar que ella misma todavía no es dicho sentido. Éste se origina en el momento en que la interacción crea y pone a disposición de los actores sociales esquemas significativos (soziale Sinnschemata), que podemos entender como junciones estables entre funciones típicas y formas de interacción típicas para realizarlas en una determinada comunidad cultural (cf. Tophinke 2001: 52-53). Salvando las distancias teóricas, es perceptible la similitud entre el concepto sociofenomenológico de los esquemas significativos y el de los cultural scripts, propuesto por la Pragmática Transcultural.

La conceptuación de la interacción comunicativa como espacio autogenético es adoptada también mediante el término autopoiesis en la Teoría del Sistema de Luhmann. La sociedad en general y la interacción comunicativa cara a cara en particular, como su forma más simple, son consideradas como sistemas operativamente cerrados, autopoiéticos, que se producen y reproducen a sí mismos por operaciones comunicativas. La participación de seres humanos (sistemas psíquicos, en la terminología de Luhmann) aparece como un prerrequisito indispensable para que la interacción tenga lugar (Luhmann 1995: 37-54).

\footnotetext{
${ }^{2}$ Cf. Tophinke (2001: 42-48) sobre semejanzas y diferencias entre los dos conceptos.
} 
Ésta, sin embargo, así como las operaciones comunicativas típicas que la generan, se establecen como un nivel sui generis de la realidad, que no puede ser equiparado y mucho menos reducido a las acciones de los actores involucrados. Luhmann (1995: 113) enfatiza esta autonomía de la comunicación con su ya famoso lema "sólo la comunicación puede comunicar" (y no el ser humano, su psique o sus acciones).

Ahora bien, si consideramos la interacción comunicativa como un espacio autogenético, se nos plantea ineludiblemente la siguiente pregunta: ¿se manifiesta la autogénesis de forma diferente en la interacción comunicativa intercultural que en la monocultural? La respuesta que les propongo es que existe tanto una diferencia respecto a los elementos que son objeto de procesos autogenéticos $\mathrm{y}$ a los niveles estructurales en que se encuentran los mismos, como a la intensidad de la autogénesis. Vamos a lo primero.

La diversidad real o potencial, en el grado que sea, de la tipicidad interactiva en los encuentros interculturales, a diferencia de los monoculturales, hace con que no podamos respaldarnos a priori en una identificación rápida, automática, posiblemente incluso inconsciente, del esquema significativo iniciado, que nos permita su mera reproducción y confirmación, a medida que el proceso interactivo va avanzando. En lugar de eso tenemos, en el caso de los encuentros interculturales, la necesidad de un mayor estado de atención y un monitoramiento más consciente por parte de los interactantes del proceso autogenético que se inicia y se desarrolla. La expectativa de una mera reproducción, por su parte, deja lugar a un proceso de producción y de atención en la producción. El esquema significativo es siempre algo potencialmente nuevo, algo a ser creado. De ello resulta que los sujetos tengan que identificar lo que está sucediendo en la interacción y tengan que identificarse a sí mismos en el proceso autogenético. Ningún elemento del proceso puede ser dejado de lado: los enunciados, las palabras que los componen, los gestos que los acompañan, las rupturas, los cambios de esquemas significativos, etc.

La profesora chilena extiende su mano para saludar al embajador iraní. Éste no le retribuye el gesto y, en lugar de ello, junta la palma de sus manos a la altura del pecho, se inclina levemente hacia delante y profiere suave "sorry". El esquema significativo de la disculpa comenta el del saludo, registrando que lo identifica como tal en su forma (extender la mano) y su función (saludar). Pero al mismo tiempo, lo rechaza como formalmente inadecuado, no aceptable. La autogénesis de la interacción intercultural nos niega el trazado, nos cambia la ruta, nos reposiciona. Nos vemos obligados a identificar el nuevo camino y a identificarnos en él.

Al mismo tiempo, con la ruptura de la expectativa de un orden conocido, en el cual el sujeto pudiera sentirse en casa, la interacción intercultural se constituye como un espacio de experiencia intensa y radical de la otredad. En cada enunciado, en cada gesto, en cada movimiento propio o del otro se manifiesta la ineludibilidad de la experiencia de la otredad. No nos podemos eximir de ella, a no ser que queramos interrumpir el proceso interactivo. Ni de la otredad del otro, ni de la otredad nuestra en nosotros y para el otro. La nuestra, la propia tan sólo se manifiesta plenamente en la interacción intercultural, es decir, cuando se nos cuestiona y relativiza la normalidad y absolutidad de nuestro ser.

Probablemente debiéramos incluir en la autogénesis de la interacción comunicativa intercultural otros elementos que se encuentran a niveles más profundos. Pienso, por ejemplo, en lo que la Etnometodología y el Análisis Conversacional Etnometodológico denominan 'mecánica de la interacción', esto es, el conjunto de mecanismos que la interacción utiliza para autogenerarse (cf. Bergmann 1994). Mecanismos estos desprovistos en sí de sentido, pero sobre los se erguen todos los sentidos de la interacción: los mecanismos que regulan los cambios de turno en la conversación, los que nos ayudan a iniciar o a finalizar interacciones, a reparar malentendidos y muchos otros. 
Deberíamos incluir también la propia creación de la intercorporeidad, como aquel espacio creado por el alineamiento de nuestros cuerpos y que nos sirve de contexto pragmático inmediato para la génesis de la interacción (Csordas 2008). Pero sugiero que lo dejemos para otro encuentro.

\section{Bibliografía}

Bergmann, Jörg R. 1994. Ethnomethodologische Konversationsanalyse, en G. Fritz y F. Hundsnurscher (eds.) Handbuch der Dialoganalyse, Tübingen, Niemeyer: 3-16.

Berger, Peter \& Thomas Luckmann. 1987. Die gesellschaftliche Konstruktion der Wirklichkeit. Eine Theorie der Wissenssoziologie, Frankfurt am Main, Fischer Taschenbuch Verlag.

Csordas, Thomas L. 2008. Intersubjectivity and Intercorporeality, Subjectivity, 22: 110-121.

Durante, Alessandro. 2011. Husserl, intersubjectivity and antropology, Antropological Theory, 10, 1: $1-20$.

Gallagher, Shaun. 2012. Phenomenology, New York, Palgrave Macmillan.

Goddard, Cliff. 2006. Ethnopragmatics - Understanding discourse in cultural context, Berlin, Mouton de Gruyter.

Kotthof, Helga y Helen Spencer-Oatey. 2007. Introduction, en H. Kotthof \& H. Spencer-Oatey (eds.) Handbook of Intercultural Communication, Berlin/New York, Mouton de Gruyter: 1-6.

Luhmann, Niklas. 1995. Soziologische Aufklärung 6. Die Soziologie und der Mensch, Opladen, Westdeutscher Verlag.

Luhmann, Niklas. 1997. Die Gesellschaft der Gesellschaft, vol. 1, Frankfurt am Main, Suhrkamp.

Schütz, Alfred. 1993. Der sinnhafte Aufbau der sozialen Welt, Frankfurt am Main, Suhrkamp.

Topkinke, Doris. 2001. Handlungstheorie, Kommunikationstheorie, Lebenswelt, en M. Haspelmath et al. (eds.) Language Typology and Language Universals, vol. 1, Walter de Gruyter, Berlin/New York: 40-62.

Waldenfels, Bernhard. 2006. Grundmotive einer Phänomenologie des Fremden, Frankfurt am Main, Suhrkamp.

Wierzbicka, Anna. 2003. Cross-Cultural Pragmatics. The Semantics of Human Interaction, Berlin/New York, Mouton de Gruyter. 